\title{
Descriptive Study of Renewable Energy System for Reactive Power Injection and AI Techniques
}

\author{
Harish Khorwal \\ M. Tech. Scholar \\ NRI Institute of Research \& Technology \\ Bhopal, India \\ harishmbmian@gmail.com
}

\author{
Mrs. Madhu Upadhyay \\ Head of Department \\ NRI Institute of Research \& Technology \\ Bhopal, India \\ madyant44@gmail.com
}

\begin{abstract}
Solar photovoltaic (PV) systems have been used primarily in the past decade. The topologies of photovoltaic networks powered by inverters are widely used to meet electricity needs and to insert renewable forms of energy into electricity grids. Managing the growing demand for electricity is currently a major problem. This paper provides a detailed overview of the topological progress of inverters connected to the photovoltaic grid, as well as the advantages and disadvantages and main characteristics of the individual inverters. The different types of inverters used in the literature in this context are presented. Reactive power is one of the auxiliary services of photovoltaic. It is recommended to supply reactive energy from the inverter to the grid in order to compensate for the reactive power in the localized grids. For proper integration into a network, coordination between the support devices used for reactive power compensation and their optimal reactive power capacity is important for the stability of the network current.
\end{abstract}

Keywords: PV, grid system, hybrid system, ANN.

\section{INTRODUCTION}

Grid-connected photovoltaic systems are electricity generation systems connected to grids. The photovoltaic solar energy generated must be processed using an inverter connected to the grid before commissioning [1]. This inverter is located between the photovoltaic solar generator and the power grid. It can be a single unit or a collection of small inverters connected to individual photovoltaic units.

Due to the lower costs of power electronic devices and advances in renewable energy technology, the energy industry is strongly encouraged to use photovoltaic solar energy and connect it to a medium or low voltage distribution network. The renewable electricity market has grown enormously in recent years. Due to lower costs, solar and wind energy are playing an increasingly important role and are competitive with fossil fuels in many countries [2].

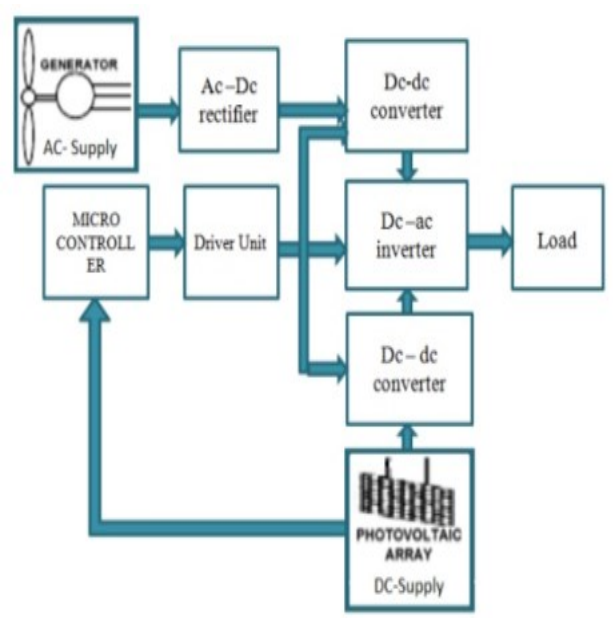

Fig. 1 Block Diagram of Hybrid Wind Solar Energy System 
It should be noted that solar PV has dominated all other forms of electricity production [3]. With this growing trend towards the use of photovoltaic solar panels, it is therefore even more important to examine the obstacles that arise to the production of energy from photovoltaic solar systems and to their export or subsequent integration into the electricity grid [4] [5]. The most important factors to consider when integrating photovoltaic solar energy into the grid are:

1. Reduction of costs during the electrical conversion phase

2. Improve the reliability of the converter used

3. Reduce harmonics in the received output current

4. Reduction in the number of switches/ components used for network integration

5. Ensure continuity of supply by providing backup energy for photovoltaic.

6. Control of active and reactive power

7. Maintaining an intermediate circuit voltage with a constant direct current thanks to an appropriate control scheme

8. Determine the maximum power point of a photovoltaic module using the MPPT (Maximum Power Point Tracking) techniques.

\section{LITERATURE REVIEW}

B. Venkatasamy et al. [1] this work mainly concerns the solar system and the inverter connected to the grid and their performance. Sun exposure is only available during the day (6 to 8 hours a day). Without solar radiation, the photovoltaic system and the inverter connected to the grid are inactive. In order to increase the use of the inverter during the day, it can also work in reactive power injection mode if photovoltaic energy is not available. This document simulates the inverter which can operate in active and reactive power injection mode. Efficient results and analyzes are carried out to improve the operation of the inverter connected to the grid used in the hybrid system.

Li-Yuan Liu et al. [2] in this article, a strategy is proposed to regulate the reactive power of the inverter connected to the grid for use in micro-grids. The proposed strategy can actively participate in voltage regulation, implement island detection, reduce the impact of island detection on energy quality and simplify the design of controls. If the distributed energy resource (DR) of the micro-grid system generates energy for the network, this can lead to an increase in the voltage of the common torque point (CCP). The proposed strategy is to use the inverter connected to the grid with reactive current control to regulate the PCC voltage. Meanwhile, reactive current disturbance is used to detect variable frequency in the island state. The experimental results also confirm the validity of the concept of planning the proposed strategy.

V. Thang Tran et al [3] This article proposes a simple structure for reactive power control (RPC) of photovoltaic modules in alternating current (AC PV). With the proposed structure, it is possible to use an inexpensive microcontroller to obtain effective control of reactive power to support the grid voltage within acceptable limits by absorbing or delivering reactive power. In addition, the sliding mode control (SMC) is used to improve the dynamic behavior of the current control and to reduce the distortion of the current harmonics. A complete demonstration of an AC PV module connected to the network via PSIM software with different operating modes is used to demonstrate the control strategy. The simulation results show that the proposal can offer good reactive power control for AC PV modules.

Leonardo B. Garcia Campanhol et al. [4] this study examines of the examination and usage of the remuneration calculations applied to a shunt dynamic force channel that utilizes three single-stage full-connect converters that share a comparable DC transport voltage. The shunt channel is applied to three-stage four-wire frameworks that evacuate the consonant current, get up to speed with deceptive power, and improve the office factor. Furthermore, remuneration for load lopsidedness is moreover performed. Two distinctive control procedures are introduced. Inside the main system, called free current control, the flows of the three-stage current source are repaid autonomously of each other by playing out consonant concealment and responsive burden power pay, for example, the three-stage framework with four wires is treated as three autonomous single-stage frameworks.

\section{Artificial Neural Network}

Artificial neural networks (ANN) or connection-oriented systems are computer systems freely inspired by the biological neural networks that structure the brain of animals. Such systems "learn" to perform tasks using 
examples, usually without being programmed with taskspecific rules. An ANN is predicated on a set of connected units or nodes called artificial neurons that vaguely shape the neurons of a biological brain. Any connection, just like the synapses of a biological brain, can transmit a sign to other neurons. A man-made neuron that receives a sign processor and may signal associated neurons [6].

In ANN implementations, the "signal" on a link may be a real and therefore the output of every neuron is calculated by a nonlinear function of the sum of its inputs. Connections are called edges. Neurons and edges generally have a weight that adapts to the progress of learning. Weight increases or decreases the signal strength on a connection. Neurons can have a threshold, so a sign is shipped as long as the general signal exceeds this threshold. As a rule, neurons are combined in layers, Different levels can bring different transformations to their inputs. The signals pass from the primary level (input level) to the last level (output level), possibly after passing through the amount several times.

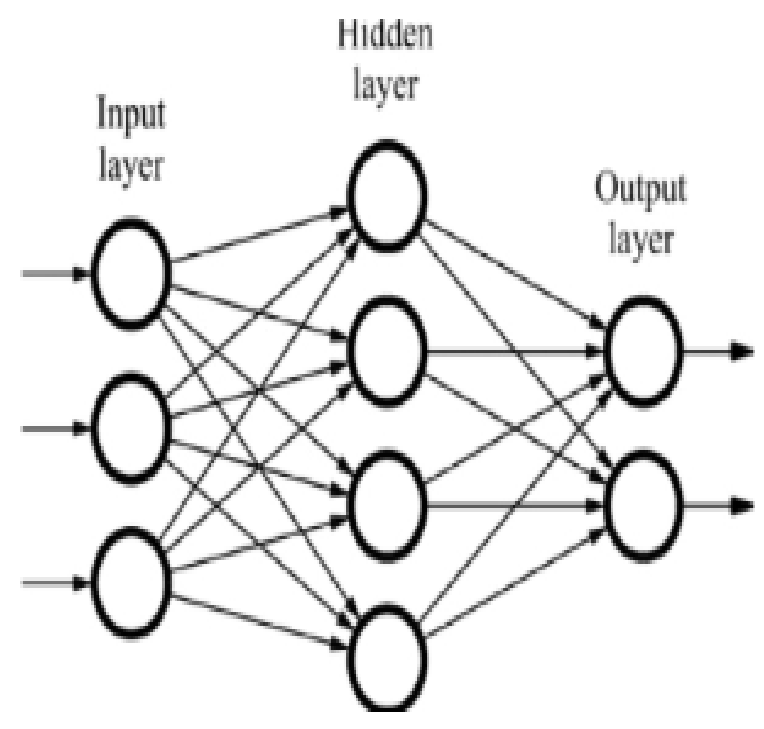

Fig. 2. Artificial Neural Network

There are several other techniques based on artificial intelligence that has been studied for optimization. Various techniques have been discussed in [7][8].

\section{STRUCTURE AND CONTROL OF GRID CONNECTED PV SYSTEM}

Single-stage PV systems do not require a DC / DC converter and MPPT control is performed via the inverter. Low cost, economic properties and high efficiency are some of the advantages of these systems thanks to their smaller converter structure. A one-tier structure is used in this study. The general structure of the PV system connected to the network is displayed. The electricity generated is transferred to the grid via photovoltaic modules with single-phase CCVSI. The MPPT controller is used to extract maximum power from the module in daylight and the RPC is used to exchange reactive power with the network in day and night modes. When using RPC, the reference current is fed into the network with a low THD and an appropriate speed [9].

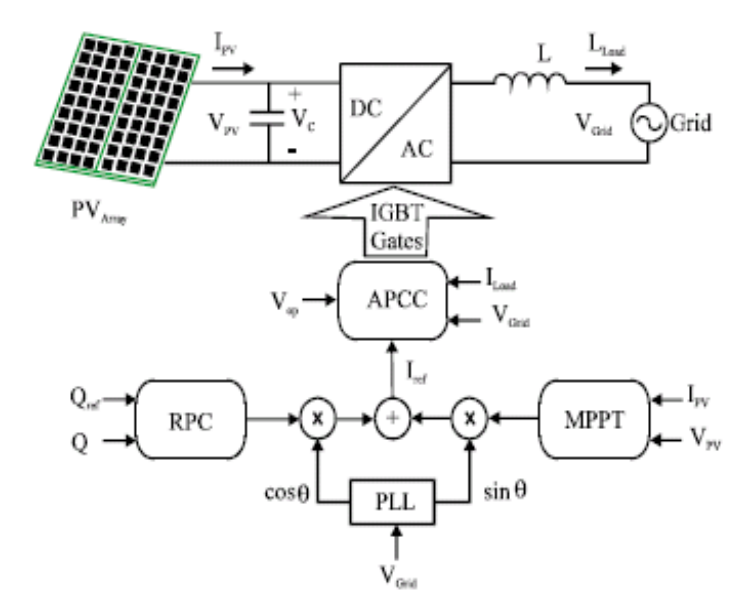

Fig. 3. Configuration and control system of proposed grid connected PV system

Solar and wind resources are alternatives to each other that have the real potential to overcome the load dilemma to some extent. However, such solutions, which are studied independently of each other, are not completely reliable because of their unstable effects. In this context, autonomous and hybrid photovoltaic systems of wind energy have proven to be a cheaper alternative to meet the energy needs of many isolated consumers around the world [10] [11].

The inverters and their outputs can be controlled for further improvement. The distortion level (THD), the output power and the voltage at the grid level must be taken into account when designing the controller for the inverter. The implementation of new electronic power devices such as compensators, filters, etc. it can also cause distortions or imbalances in the system. This 
change must be overcome when designing a control system. Since the trend supports the use of renewable and clean energy sources, the system can be further integrated with other sources such as fuel cell technology or geothermal stations, etc.

\section{CONCLUSION}

In this paper, a hybrid system with solar power generation is used for analysis. The need for reactive energy production in the photovoltaic inverter was discussed. The control strategy for generating reactive energy in the grid inverter was discussed. The Simulink model of the photovoltaic system connected to the network is developed with MATLAB. The inverter operating in active and reactive energy production mode has been simulated. The different active and reactive power measurements were used for the analysis. Reactive power compensation must be performed in a solar power plant to improve the efficient use of the system.

\section{REFERENCES}

[1] B. Venkatasamy, L. Kalaivani "Performance Analysis of GridTie Inverter for Reactive Power Injection Mode in Hybrid Wind Solar Energy System" DOI: 10.1109/ICOEI.2018.8553869

[2] Li-Yuan Liu, Jun-Ting Gao, \&Kuo-Yuan Lo. (2017). "A reactive power control strategy of the grid-connected inverter for microgrid application”. 2017 IEEE 3rd International Future Energy Electronics Conference and ECCE Asia doi:10.1109/ifeec.2017.7992134

[3] Tran, V. T., Sutanto, D., \& Muttaqi, K. M. (2015). Simple structure for reactive power control of AC photovoltaic modules. 2015 Australasian Universities Power Engineering Conference doi:10.1109/aupec.2015.7324884

[4] Garcia Campanhol, L. B., Oliveira da Silva, S. A., \&Goedtel, A. (2014). Application of shunt active power filter for harmonic reduction and reactive power compensation in three-phase fourwire systems. IET Power Electronics, 7(11), 28252836. doi:10.1049/iet-pel.2014.0027

[5] Tran, D. Sutanto\& K. M. Muttaqi, "Simple structure for reactive power control of AC photovoltaic modules," in Power Engineering Conference (AUPEC), 2015 Australasian Universities, 2015, pp. 1-6.

[6] Y. Bae, T.-K. Vu, and R.-Y. Kim, "Implemental control strategy forgrid stabilization of grid-connected PV system based on German grid code in symmetrical low-to-medium voltage network," IEEE Trans. Energy Convers., vol. 28, no. 3, pp. 619631, Sep. 2013.

[7] Y. Chen, A. Luo, Z. Shuai, and S. Xie, "Robust predictive dualloop control strategy with reactive power compensation for single-phase grid-connected distributed generation system," IET Power Electron.,vol.6, no. 7, pp. 1320-1328, Aug. 2013.

[8] M. Islam, S. Mekhilef, and M. Hasan, "Single phase transformer less inverter topologies for grid-tied photovoltaic system: A review, Renew. Sustainable Energy Rev., vol. 45, pp. 69-86, 2015.

[9] Zhang, K. Sun, Y. Xing, and M. Xing, "H6 transformer less full bridge PV grid-tied inverters," IEEE Trans. Power Electron., vol. 29,no. 3, pp. 1229-1238, Mar. 2014.

[10] J. Hu, L. Shang, Y. He, and Z. Q. Zhu, "Direct active and reactive power regulation of grid-connected DC/AC converters using sliding mode control approach," IEEE Trans. Power Electron., vol. 26, no. 1,pp. 210-222, Jan. 2011.

[11] Ramesh Bansal, T. S. Bhatti, D.P. Kothari "artificial intelligence techniques for reactive power/voltage control in power systems: a review", International Journal of Power and Energy Systems, 2003 